Moroccan J. of Pure and Appl. Anal. (MJPAA)

Volume 7(1), 2021, Pages 43-49

ISSN: Online 2351-8227 - Print 2605-6364

DOI: $10.2478 / \mathrm{mjpaa}-2021-0005$

\title{
On a chain of reproducing kernel Cartan subalgebras
}

\author{
Anoh Yannick Kraidi ${ }^{1}$ and Kinvi Kangni ${ }^{2}$
}

AвsтRAст. Let $\mathfrak{g}$ be a semisimple Lie algebra, $\mathfrak{j}$ a Cartan subalgebra of $\mathfrak{g}, \mathfrak{j}^{*}$, the dual of $\mathfrak{j}, \mathfrak{j}^{\vee}$ the bidual of $\mathfrak{j}$ and $B(.,$.$) the restriction to \mathfrak{j}$ of the Killing form of $\mathfrak{g}$. In this work, we will construct a chain of reproducing kernel Cartan subalgebras ordered by inclusion.

Mathematics Subject Classification (2020). 17B20, 46E22, 17B22.

Key words and phrases. Cartan subalgebra, Root systems, Reproducing kernel.

\section{Introduction}

Let $E$ be a set. A reproducing kernel Hilbert space $H$ on $E$ is a Hilbert space of functions from $E$ with values in $\mathbb{C}$, for which the evaluation function in each point of $E$ is continuous (see $[1,2,5,7,8])$. Thanks to Riesz - Fréchet theorem, one can deduce the existence of a kernel $K: E \times E \rightarrow \mathbb{C}$ such that for all $x \in E$,

$$
f(x)=(f, K(., x))_{H}, \text { for all } f \in H .
$$

The kernel verifies

$$
K(x, y)=K_{y}(x)=(K(., y), K(., x))_{H} \text { for all } x, y \in E .
$$

Received : 23 March 2020 - Accepted: 30 October 2020.

(C)The Author(s) 2020. This article is published with open access by Sidi Mohamed Ben Abdallah University.

${ }^{1}$ UFR Mathmatiques et Informatique, Universit Flix Houphouet-Boigny de Cocody-Abidjan; 25 BP 1245 Abidjan 25 e-mail: Kayanoh2000@yahoo.fr (Corresponding Author).

${ }^{2}$ UFR Mathmatiques et Informatique, Universit Flix Houphouet-Boigny de Cocody-Abidjan; 22 BP 1214 Abidjan 22 e-mail: kinvi.kangni@univ-fhb.edu.ci. 
We have another approach of the reproducing kernel Hilbert space in the case of linear transformations which is given by the theorem of Aronszajn.

Furthermore, the theorem of Aronszajn (see $[10,13])$ ensures that for every positive definite kernel $K$, there exists a unique Hilbert space $V$ of complex functions on $E$ where $K$ is the reproducing kernel. That is, for all $x \in E$,

$$
f(x)=(f, K(., x))_{V}, \text { for all } f \in V .
$$

Let $\mathfrak{g}$ be a semisimple Lie algebra and $\mathfrak{j}$ a Cartan subalgebra of $\mathfrak{g}, \mathfrak{j}^{*}$ the dual of $\mathfrak{j}$ and $B(.,$. the restriction to $\mathfrak{j}$ of the Killing form of $\mathfrak{g}$ (see $[4,6])$.

Let's consider the Hilbert space $j^{*}$ with the scalar product $(., .)_{j *}$ which is the Killing form on $\mathfrak{j}^{*}, \mathcal{F}(E)$ the set of complex functions defined on $E, h$ a function on $E$ with values in $\mathfrak{j}^{*}$ defined by $h(p)=h_{p}, L$ the map from $\mathfrak{j}^{*}$ to $\mathcal{F}(E)$ such that for all $p \in E, f \in \mathfrak{j}^{*}$ with $\tilde{f}=L f$,

$\tilde{f}(p)=(L f)(p)=\left(f, h_{p}\right)_{\mathfrak{j}^{*}}=f\left(H_{p}\right)=B\left(F, H_{p}\right)$ where $H_{p}, F \in \mathfrak{j}$ (see [15] p.3).

Let's consider the kernel $K$ defined by:

$$
K(p, q)=\left(h_{q}, h_{p}\right)_{j^{*}}=L\left(h_{q}\right)(p)=B\left(H_{q}, H_{p}\right) \text { for all } p, q \in E .
$$

Since $B$ is non-degenerate (See $[6,9,12]$ ), the kernel $K$ is positive definite. Then, by the theorem of Aronszajn, there exists a unique Hilbert space where $K$ is the kernel, denoted by $h_{K}$ such that for all $p \in E$,

$$
g(p)=(g, K(., p))_{h_{K}} \text { for all } g \in h_{K} .
$$

Let $R(L)$ be the range of $L$. We introduce an inner product in $R(L)$ (see [3]) induced by the norm:

$$
\|\tilde{f}\|_{R(L)}=\inf \left\{\|f\|_{j^{*}} ; \tilde{f}=L f\right\} .
$$

$R(L)$ is a Hilbert space verifying the forgoing assumptions (see [10, Theorem 1 p.21]). By uniqueness of the reproducing kernel Hilbert space, we have $R(L)=h_{K}$ and

$$
(L f)(p)=\tilde{f}(p)=(\tilde{f}, K(., p))_{h_{K}} \text {, for all } f \in j^{*} .
$$

Considering the bidual $\mathfrak{j}^{\vee}$ of the Cartan subalgebra $\mathfrak{j}, \mathfrak{j}^{\vee}$ is the reproducing kernel Cartan subalgebra (RKCS) when $E$ is identified to $\mathfrak{j}^{*}$. The kernel of $\mathfrak{j}^{\vee}$ is defined by $K(\alpha, \beta)=\left(h_{\beta}, h_{\alpha}\right)_{j^{*}}$ for all $\alpha, \beta \in \mathfrak{j}^{*}$.

In fact, for all $a \in \mathfrak{j}^{\vee}$, there exists a unique $a^{*} \in \mathfrak{j}^{*}$ such that $a\left(b^{*}\right)=B\left(a^{*}, b^{*}\right)$ for all $b^{*} \in j^{*}($ see $[12,15])$. Then, we have

$$
a\left(b^{*}\right)=\left(a, K\left(., b^{*}\right)\right)_{\mathfrak{j} \vee} \text { for all } b^{*} \in \mathfrak{j}^{*} \text {, where } K\left(a^{*}, b^{*}\right)=(b, a)_{\mathrm{j} \vee} .
$$

Note that $B$ is strictly positive definite on $j^{*} \times \mathfrak{j}^{*}$. Let's consider the real semisimple Lie algebra $\mathfrak{g}^{\sharp}$ equipped with the involution $\theta . \mathfrak{g}^{\sharp}$ is a Hilbert space with the scalar product $(., .)_{\theta}$. This Killing form leads to a map $p_{\theta}(x, y)=(x, y)_{\theta}=-B(x, \theta(y))$, for all $x, y \in \mathfrak{g}^{\sharp}$ (see [15]). Therefore, we define the following map: $K_{p_{\theta}}(x, y)=(x, y)_{\theta} \cdot K_{p_{\theta}}$ is positive definite, thus by the theorem of Aronszajn, there exists a unique Hilbert space $H_{\theta}$ such that for all $x \in \mathfrak{g}^{\sharp}$, $K_{p_{\theta}}(., x) \in H_{\theta}$ and

$$
f(x)=\left(f, K_{p_{\theta}}(., x)\right)_{H_{\theta}} \text {, for all } f \in H_{\theta} .
$$

For all $x, y \in \mathfrak{g}^{\sharp}, K_{p_{\theta}}(x, y)=K_{p_{\theta}}(y)(x)=\left(K_{p_{\theta}}(., y), K_{p_{\theta}}(., x)\right)_{H_{\theta}}$.

This Hilbert space will be called the Hilbert space of type $\theta$ and its kernel is of type $\theta$. 
In this work, we will prove some results about the Hilbert space $H_{\theta}$ and construct a chain of reproducing kernel Cartan subalgebras ordered by inclusion (as in [10] p.52).

\section{Chain by inclusion of reproducing kernel Cartan subalgebras}

Let $\mathfrak{g}$ be a semisimple Lie algebra and $\mathfrak{j}$ a Cartan subalgebra of $\mathfrak{g}, \mathfrak{j}^{*}$ the dual of $\mathfrak{j}$ and $\mathfrak{j}^{\vee}$, the bidual of $\mathfrak{j}$.

Let $\mathfrak{g}^{\sharp}$ be a real semisimple Lie algebra with an involution $\theta$. We consider the Hilbert space of type $\theta$ and its kernel of type $\theta$. Let $U$ be a representation of $\mathfrak{g}^{\sharp}$ on $H_{\theta}$. We consider for $g, x, y \in$ $\mathfrak{g}^{\sharp},\left(U_{g}^{1}\right)^{*} K_{p_{\theta}}(x, y)$ which means that the representation is applied to $K_{p_{\theta}}(x, y)$ as function of the first variable $x$. The function obtained will be considered as function of $x$ but it will depend on $y$ which will act as a parameter, where $\left(U_{g}^{1}\right)^{*}$ is the adjoint of $U_{g}^{1}$. We have

Theorem 2.1. The function defined on $\mathfrak{g}^{\sharp} \times \mathfrak{g}^{\sharp}$ by $\Lambda_{g}^{U^{1}}(y, z)=\left(U_{g}^{1}\right)^{*} K_{p_{\theta}}(y, z)$ for all $y, z \in \mathfrak{g}^{\sharp}$ is positive definite if and only if $U_{g}^{1}$ is positive.

For all $g \in \mathfrak{g}^{\sharp}$, if $U_{g}^{1}$ is positive then $\Lambda_{g}^{U^{1}}$ is a positive definite kernel corresponding to $U_{g}^{1}$ and the reproducing kernel space is denoted by $H_{\Lambda^{1}}$. The kernel corresponding to $\left(U_{g}^{1}\right)^{*}$, the adjoint of $U_{g}^{1}$ is $\left(\Lambda_{g}^{U^{1}}\right)^{*}(y, z)=\overline{\left(\Lambda_{g}^{U^{1}}\right)(z, y)}$ for all $y, z \in \mathfrak{g}^{\sharp}$ and the kernel corresponding to $U_{g}^{1} \circ U_{g^{\prime}}^{1}$ is

$$
\left(\Lambda_{g g^{\prime}}^{U^{1}}\right)(y, z)=\left(\Lambda_{g}^{U^{1}}(., z), \overline{\Lambda_{g^{\prime}}^{U^{1}}(y, .)}\right)_{H_{\theta}} \text { for all } y, z, g, g^{\prime} \in \mathfrak{g}^{\sharp} .
$$

Proof. For all $g \in \mathfrak{g}^{\sharp}$, let's assume that $U_{g}^{1}$ is positive.

Let us find the kernel $\left(\Lambda_{g}^{U^{1}}\right)^{*}$ corresponding to the adjoint operator $\left(U_{g}^{1}\right)^{*}$. For $y, z, g \in \mathfrak{g}^{\sharp}$, $\left(\left(U_{g}^{1}\right)^{*}\right)^{*}=U_{g}^{1}$, and

$$
\begin{aligned}
& \left(U_{g}^{1} K_{p_{\theta}}(., z), K_{p_{\theta}}(., y)\right)_{H_{\theta}}=\underline{\left(K_{p_{\theta}}(., z),\left(\left(U_{g}^{1}\right)^{*} K_{p_{\theta}}(., y)\right)_{H_{\theta}}\right.} \\
& \begin{array}{lll}
\left(\left(\Lambda_{g}^{U^{1}}\right)^{*}(., z), K_{p_{\theta}}(., y)\right)_{H_{\theta}} & = & \frac{\left(\left(\Lambda_{g}^{U^{1}}\right)(., y), K_{p_{\theta}}(., z)\right)_{H_{\theta}}}{\text { then }^{\prime}\left(\Lambda_{g}^{U^{1}}\right)^{*}(y, z)}= \\
\Lambda_{g}^{U^{1}}(z, y) .
\end{array}
\end{aligned}
$$

Consider $y_{1}, y_{2}, \ldots, y_{n} \in \mathfrak{g}^{\sharp}, c_{1}, c_{2}, \ldots, c_{n} \in \mathbb{C}$ with $n \in \mathbb{N}$.

For $f=\sum_{k=1}^{n} c_{k} K_{p_{\theta}}\left(., y_{k}\right)$,

$$
\begin{array}{rlrl}
\sum_{i=1}^{n} \sum_{j=1}^{n} c_{i} \overline{c_{j}}\left(U_{g}^{1} K_{p_{\theta}}\left(., y_{i}\right), K_{p_{\theta}}\left(., y_{j}\right)\right)_{H_{\theta}} & = & \sum_{i=1}^{n} \sum_{j=1}^{n} c_{i} \overline{c_{j}}\left(\left(\Lambda_{g}^{U^{1}}\right)^{*}\left(., y_{i}\right), K_{p_{\theta}}\left(., y_{j}\right)\right)_{H_{\theta}} \\
& = & \sum_{i=1}^{n} \sum_{j=1}^{n}\left(\Lambda_{g}^{U^{1}}\right) *\left(y_{j}, y_{i}\right) c_{i} \overline{c_{j}} \\
& = & & \sum_{i=1}^{n} \sum_{j=1}^{n} \overline{\Lambda_{g}^{U^{1}}}\left(y_{i}, y_{j}\right) c_{i} \overline{c_{j}} \\
& \geq & & 0 .
\end{array}
$$

We proved that $\overline{\Lambda_{g}^{U^{1}}}$ is positive definite thus $\Lambda_{g}^{U^{1}}$ is positive definite. 
For all $g \in \mathfrak{g}^{\sharp}$, let's assume that $\Lambda_{g}^{U^{1}}$ is positive definite, we notice that $\left(U_{g}^{1} f, f\right) \geq 0$ for all $f$ of the form $f=\sum_{k=1}^{n} c_{k} K_{p_{\theta}}\left(., y_{k}\right)$. As these functions form a dense set in $H_{\theta}$, every $f$ may be approximated by them and we get $\left(U_{g}^{1} f, f\right) \geq 0$ by approaching the limit.

We consider the adjoint operator $\left(U_{g}^{1}\right)^{*}$ with $g \in \mathfrak{g}^{\sharp}$. For $f, k \in H_{\theta},\left(\left(U_{g}^{1}\right)^{*} f, k\right)_{H_{\theta}}=\left(f, U_{g}^{1} k\right)_{H_{\theta}}$ and for $x, y \in \mathfrak{g}^{\sharp}, \Lambda_{g}^{U^{1}}(x, y)=\left(U_{g}^{1}\right)^{*} K_{p_{\theta}}(x, y)$;

$\Lambda_{g}^{U^{1}}$ is a function of two points $x$ and $y$.

If we take any $f \in H_{\theta}$ and $y \in \mathfrak{g}^{\sharp}$, then we get

$$
\begin{array}{rlc}
\left(f, \Lambda_{g}^{U^{1}}(., y)\right)_{H_{\theta}} & = & \left(f,\left(U_{g}^{1}\right)^{*} K_{p_{\theta}}(., y)\right)_{H_{\theta}} \\
& = & \left(U_{g}^{1} f, K_{p_{\theta}}(., y)\right)_{H_{\theta}} \\
& = & U_{g}^{1} f(y) .
\end{array}
$$

Thus, $U_{g}^{1} f(y)=\left(f, \Lambda_{g}^{U^{1}}(., y)\right)_{H_{\theta}}$. We deduce that each operator $U_{g}^{1}$ corresponds to a kernel $\Lambda_{g}^{U^{1}}(x, y)$ for which every $y$, as function of $x$ belongs to $H_{\theta}$.

We shall now give the kernel corresponding to the composition $U_{g}^{1} \circ U_{g^{\prime}}^{1}$ with $g, g^{\prime} \in \mathfrak{g}^{\sharp}$. Since $\left(U_{g}^{1} \circ U_{g^{\prime}}^{1}\right)^{*}=\left(U_{g^{\prime}}^{1}\right)^{*} \circ\left(U_{g}^{1}\right)^{*}$, for $y, z \in \mathfrak{g}^{\sharp}$,

$$
\begin{aligned}
& \left(\Lambda_{g g^{\prime}}^{U^{1}}\right)(y, z)=\left(U_{g}^{1} \circ U_{g^{\prime}}^{1}\right)^{*} K_{p_{\theta}}(y, z) \\
& =\left(U_{g^{\prime}}^{1}\right)^{*} \circ\left(U_{g}^{1}\right)^{*} K_{p_{\theta}}(y, z) \\
& =\left(U_{g^{\prime}}^{1}\right)^{*} \Lambda_{g}^{U^{1}}(y, z) \\
& =\left(\Lambda_{g}^{U^{1}}(., z),\left(\underline{\left.\Lambda_{g^{\prime}}^{U^{1}}\right)^{*}(., y)}\right)_{H_{\theta}}\right. \\
& =\left(\Lambda_{g}^{U^{1}}(., z),\left(\overline{\left.\Lambda_{g^{\prime}}^{U^{1}}\right)(y, .)}\right) H_{\theta} .\right.
\end{aligned}
$$

Then, $\left(\Lambda_{g g^{\prime}}^{U^{1}}\right)^{*}(y, z)=\left(\Lambda_{g}^{U^{1}}(., z), \overline{\Lambda_{g^{\prime}}^{U^{1}}(y, .)}\right)_{H_{\theta}}$.

Theorem 2.2. If we consider the representation $U$ of $\mathfrak{g}^{\sharp}$ on $H_{\theta}$ and the kernel $\Lambda_{g}^{U^{1}}(x, y)$ with $g, x, y \in \mathfrak{g}^{\sharp}$, then the necessary and sufficient condition that this kernel corresponds to a bounded operator $U_{g}^{1}$ with lower bound $m$ and upper bound $M$ is: $m K_{p_{\theta}} \leq \Lambda_{g}^{U^{1}} \leq M K_{p_{\theta}}$.

Proof. We consider $g \in \mathfrak{g}^{\sharp}$ and we suppose that $U_{g}^{1}$ admits bounds not less than $m$ and not greater than $M$, that means that we have

$$
m(f, f)_{H_{\theta}} \leq\left(U_{g}^{1} f, f\right)_{H_{\theta}} \leq M(f, f)_{H_{\theta}} \text {, for all } f \in H_{\theta} .
$$

It follows that $\left(\left(U_{g}^{1}-m I\right) f, f\right)_{H_{\theta}} \geq 0$ and $\left(\left(M I-U_{g}^{1}\right) f, f\right)_{H_{\theta}} \geq 0$; that is, the operators $U_{g}^{1}-m I$ and $M I-U_{g}^{1}$ are positive. By the previous results, we deduce that $\Lambda_{g}^{U^{1}}-m K_{p_{\theta}}$ and $M K_{p_{\theta}}-\Lambda_{g}^{U^{1}}$ are positive definite.

Suppose that there exists some constants $m$ and $M$ such that

$$
m K_{p_{\theta}}(x, y) \leq \Lambda_{g}^{U^{1}}(x, y) \leq M K_{p_{\theta}}(x, y), \text { for all } x, y \in \mathfrak{g}^{\sharp} .
$$


Then, $0 \leq \frac{1}{M-m}\left(\Lambda_{g}^{U^{1}}(x, y)-m K_{p_{\theta}}(x, y)\right) \leq K_{p_{\theta}}(x, y)$, for all $x, y \in \mathfrak{g}^{\sharp}$.

We deduce that the kernel $K_{1}=\frac{1}{M-m}\left(\Lambda_{g}^{U^{1}}(x, y)-m K_{p_{\theta}}(x, y)\right)$ is positive definite and $K_{1}(x, y) \leq$ $K_{p_{\theta}}(x, y)$ for all $x, y \in \mathfrak{g}^{\sharp}$, then there exists a

reproducing kernel Hilbert space $H_{1}$ where $K_{1}$ is the kernel such that

$H_{1} \subset H_{\theta}$ and $\left\|f_{1}\right\|_{H_{1}} \geq\left\|f_{1}\right\|_{H_{\theta}}$ for $f_{1} \in H_{1}$.

We introduce for all $x, y \in \mathfrak{g}^{\sharp}$, the operator $\left(\left(U_{g}^{\prime}\right)^{1}\right) f(y)=\left(f, K_{1}(., y)\right)_{H_{\theta}}$ obtained from another representation $U^{\prime}$ on $\mathfrak{g}^{\sharp}$; the operator is positive in $H_{\theta}$ with a bound not greater than 1 (see [2, Theorem III p.356]), that means $0 \leq\left(\left(U_{g}^{\prime}\right)^{1} f, f\right)_{H_{\theta}} \leq(f, f)_{H_{\theta}}$. Since $\left(U_{g}^{\prime}\right)^{1}$ corresponds to $K_{1}$, then $\Lambda_{g}^{U^{1}}=(M-m) K_{1}+m K_{p_{\theta}}$ corresponds to $U_{g}^{1}=(M-m)\left(U_{g}^{\prime}\right)^{1}+m I$ and for all $f \in H_{\theta}$, the last inequalities give:

$$
\begin{gathered}
m(f, f)_{H_{\theta}} \leq(m I f, f)_{H_{\theta}}+\left((M-m)\left(U_{g}^{\prime}\right)^{1} f, f\right)_{H_{\theta}} \leq M(f, f)_{H_{\theta}} \\
\quad \text { and } \\
m(f, f)_{H_{\theta}} \leq\left(U_{g}^{1} f, f\right)_{H_{\theta}} \leq M(f, f)_{H_{\theta}} .
\end{gathered}
$$

Let $T$ be a bounded linear operator from the reproducing kernel space $j^{\vee}$ into $H_{\Lambda^{u}}$. Let's introduce (see [11]) the following inner products:

$$
\begin{aligned}
& \left(\tilde{\beta}_{1}, \tilde{\beta}_{2}\right)_{j^{\vee}\left[H_{\Lambda} U^{1}\right]^{+}}=\left(\tilde{\beta}_{1}, \tilde{\beta}_{2}\right)_{j^{\vee} \vee}+\left(T \tilde{\beta}_{1}, T \tilde{\beta}_{2}\right)_{H_{\Lambda} U^{1}} \\
& \text { and } \left.\tilde{\beta}_{1}, \tilde{\beta}_{2}\right)_{j^{\vee}\left[H_{\Lambda^{1}}\right]^{-}}=\left(\tilde{\beta}_{1}, \tilde{\beta}_{2}\right)_{\mathfrak{j}} \vee-\left(T \tilde{\beta}_{1}, T \tilde{\beta}_{2}\right)_{H_{\Lambda^{1}}} .
\end{aligned}
$$

We shall construct the reproducing kernels for $\mathfrak{j}^{\vee}\left[H_{\Lambda^{U^{1}}}\right]^{+}$and $\mathfrak{j}^{\vee}\left[H_{\Lambda^{u^{1}}}\right]^{-}$such that

$$
\mathfrak{j}^{\vee}\left[H_{\Lambda^{U^{1}}}\right]^{+} \subset \mathfrak{j}^{\vee} \subset \mathfrak{j}^{\vee}\left[H_{\Lambda^{U^{1}}}\right]^{-} \text {. }
$$

We consider the linear map defined by $I: H_{\Lambda^{u^{1}}} \longrightarrow \mathcal{F}\left(\mathfrak{j}^{*}\right)$

such that for all $\beta \in \mathfrak{j}^{*}, \check{a}(\beta)=(a, T(K(., \beta)))_{H_{\Lambda} U^{1}}$.

Theorem 2.3. For $\beta \in \mathfrak{j}^{*}, \check{a}=T^{*} a$ and $\|\check{a}\|_{\mathfrak{j}^{\vee}}^{2}=\left(a, T(a, T K(., \beta))_{H_{\Lambda^{1}}}\right)_{H_{\Lambda} u^{1}}$. Furthermore, the following conditions are equivalent:

$1-K(\alpha, \beta) \geq(T K(., \beta), T K(., \alpha))_{H_{\Lambda} U^{1}}$.

2- $\|T\| \leq 1$.

3- $\|\check{a}\|_{j \vee} \leq\|a\|_{H_{\Lambda} U^{1}}$.

Proof. Let be $\beta \in \mathfrak{j}^{*}$ and $a \in H_{\Lambda^{u^{1}}}$. $\check{a}(\beta)=(a, T(K(., \beta)))_{H_{\Lambda^{u}}}=\left(T^{*} a, K(., \beta)\right)_{\mathfrak{j}^{\vee}}$. Since $\check{a} \in \mathfrak{j}^{\vee}, \check{a}(\beta)=(\check{a}, K(., \beta))_{\mathfrak{j} \vee}$, then $\check{a}=T^{*} a$ and

$$
\begin{array}{rlc}
\|\check{a}\|_{j^{\vee}}^{2} & = & (\check{a}, \check{a})_{\mathfrak{j} \vee} \\
& =\left(T^{*} a,(a, T(K(., .)))_{H_{\Lambda^{U^{1}}}}\right)_{H_{\Lambda} U^{1}} \\
& = & \left(a, T(a, T K(., .))_{H_{\Lambda} U^{1}}\right)_{H_{\Lambda^{1}}} .
\end{array}
$$

Now, we show the equivalences. 
$1 \Longrightarrow 3$.

We consider the following positive definite kernel $K_{T}$ defined by:

$K_{T}(\alpha, \beta)=(T K(., \beta), T K(., \alpha))_{H_{\Lambda^{1}}}$ on $\mathfrak{j}^{*} \times \mathfrak{j}^{*}$, the reproducing kernel space where $K_{T}$ is the kernel will be denoted by $h_{K_{T}}$. Since the range of $I$ verifies $R(I)=h_{K_{T}}$, we obtain $\|\check{a}\|_{h_{K_{T}}} \leq\|a\|_{H_{\Lambda} U^{1}}$. If 1 is true, we have $\|\check{a}\|_{j \vee} \leq\|\check{a}\|_{h_{K_{T}}}$ because we know that for two positive definite kernels $K^{(1)}(p, q)$ and $K^{(2)}(p, q)$ defined on any set $E \times E$,

if $K^{(1)}(p, q) \leq K^{(2)}(p, q)$ on $E \times E$, then for the reproducing kernels Hilbert spaces $H_{K^{(1)}}$ and $H_{K^{(2)}}$, we have $\|f\|_{H_{K^{(1)}}} \geq\|f\|_{H_{K^{(2)}}}$ for any $f \in H_{K^{(1)}}$ (see [2, Theorem I p.354]). So, we get $\|\check{a}\|_{j \vee} \leq\|\check{a}\|_{h_{K_{T}}} \leq\|a\|_{H_{\Lambda} U^{1}}$.

$3 \Longrightarrow 2$

Assume 3. For $a \in H_{\Lambda^{U^{1}}}$ and $\check{a} \in \mathfrak{j}^{\vee}$ such that $\|\check{a}\|_{j^{\vee}} \leq\|a\|_{H_{\Lambda^{U^{1}}}}$, we get $\|T\| \leq 1$ (See [3]).

\section{$2 \Longrightarrow 1$}

Assume 2. We know that for two positive definite kernels $K^{(1)}(p, q)$ and $K^{(2)}(p, q)$ on $E \times E$, $H_{K^{(1)}} \subset H_{K^{(2)}}$ if and only if there exists a positive constant $\gamma$, such that $K^{(1)}(p, q) \leq \gamma^{2} K^{(2)}(p, q)$ and the minimum of such $\gamma$ coincides with the norm of the inclusion map from $H_{K^{(1)}}$ into $H_{K^{(2)}}$ (see [14, Theorem 5.1 p.66]). By this result, we have that the map $\check{a}=T^{*} a$ is into $j^{\vee}$ and onto $j^{\vee}$ if and only if $K_{T}(\alpha, \beta)=(T K(., \beta), T K(., \alpha))_{H_{\Lambda} u^{1}} \leq K(\alpha, \beta)$ (see [10, Lemna 3 p.51]), hence 1 is proved.

For $\check{a} \in \mathfrak{j}^{\vee}\left[H_{\Lambda^{U^{1}}}\right]^{+},\|\check{a}\|_{\mathfrak{j}^{\vee}\left[H_{\Lambda^{\prime} u^{1}}\right]^{+}} \geq\|\check{a}\|_{j^{\vee}}$, then there exists a reproducing kernel $K_{H_{\Lambda} U^{1}}^{+}$for the space $\mathfrak{j}^{\vee}\left[H_{\Lambda^{U^{1}}}\right]^{+}$such that $K_{H_{\Lambda^{1}}}^{+}(\alpha, \beta) \leq K(\alpha, \beta)$ on $\mathfrak{j}^{*} \times \mathfrak{j}^{*}$ (see [2, Theorem II p.355]). Let's consider the kernel $K$ defined on $\mathfrak{j}^{*} \times \mathfrak{j}^{*}$ by $K(\alpha, \beta)=\left(h_{\beta}, h_{\alpha}\right)_{\mathfrak{j}^{*}}=(K(., \beta), K(., \alpha))_{\mathfrak{j}^{\vee}}$.

By the following theorem, we construct the reproducing kernel $K_{H_{\Lambda} U^{1}}^{+}(\alpha, \beta)$ using $K(\alpha, \beta)$ for $\alpha, \beta \in \mathfrak{j}^{*}$.

Theorem 2.4. Given that $\left\{\tilde{K}(., \beta), \beta \in j^{*}\right\} \subset j^{\vee}$, the set of functions satisfying the following equation

$$
K(\alpha, \beta)=\tilde{K}(\alpha, \beta)+(T \tilde{K}(., \beta), T K(., \alpha))_{H_{\Lambda} u^{1}}
$$

Then the kernel $\tilde{K}(\alpha, \beta)$ is a solution of the functional equation (1) if and only if $\tilde{K}(\alpha, \beta)$ is the reproducing kernel of $\mathfrak{j}^{\vee}\left[H_{\Lambda^{u^{1}}}\right]^{+}$.

Proof. For any $\gamma, \alpha \in \mathfrak{j}^{*}$, we have

$$
\begin{gathered}
(K(., \gamma), K(., \alpha))_{j} \vee=(\tilde{K}(., \gamma), K(., \alpha))_{j} \vee+(T \tilde{K}(., \gamma), T \tilde{K}(., \alpha))_{j^{*}} \\
\Leftrightarrow K(., \gamma)=\tilde{K}(., \gamma)+T^{*} T \tilde{K}(., \gamma) .
\end{gathered}
$$

For all $\tilde{\beta} \in \mathfrak{j}^{\vee}$,

$$
(\tilde{\beta}, K(., \gamma))_{j} \vee=(\tilde{\beta}, \tilde{K}(., \gamma))_{j} \vee+\left(\tilde{\beta}, T^{*} T \tilde{K}(., \gamma)\right)_{j} \vee
$$




$$
\left.\Leftrightarrow \tilde{\beta}(\gamma)=(\tilde{\beta}, \tilde{K}(., \gamma))_{\mathfrak{j}^{\vee}\left[H_{\Lambda} U^{1}\right.}\right]^{+} \text {. }
$$

If for any $\tilde{\beta} \in \mathfrak{j}^{\vee},\|\tilde{\beta}\|_{j^{\vee}} \geq\|T \tilde{\beta}\|_{H_{\Lambda^{\prime} U^{1}}}$ with the equality if and only if $\tilde{\beta}=0$, then we can introduce the pre-Hilbert space $H^{\prime}$ equipped with the inner product

$$
\left(\tilde{\beta}_{1}, \tilde{\beta}_{2}\right)_{H^{\prime}}=\left(\tilde{\beta}_{1}, \tilde{\beta}_{2}\right)_{\mathrm{j} v}-\left(T \tilde{\beta}_{1}, T \tilde{\beta}_{2}\right)_{H_{\Lambda} U^{1}} \text {. }
$$

For any fixed $\alpha \in \mathfrak{j}^{*}, \tilde{\beta}(\alpha)$ the evaluation of $\tilde{\beta}$ at point $\alpha$ is bounded on $H^{\prime}$ and for any Cauchy sequence $\left\{\tilde{\beta}_{n}\right\}$ in $H^{\prime}$, the condition $\tilde{\beta}_{n} \longrightarrow 0$ on $j^{*}$ implies $\left\|\tilde{\beta}_{n}\right\|_{H^{\prime}} \longrightarrow 0$. By the completion, $\overline{H^{\prime}}$ is unique and admits the reproducing kernel.

We shall denote the completion of $H^{\prime}$ by $j^{\vee}\left[H_{\Lambda^{U^{1}}}\right]^{-}$, if the conditions above are satisfied.

Then, for any $\tilde{\beta} \in \mathfrak{j}^{\vee},\|\tilde{\beta}\|_{\mathfrak{j}^{\vee}\left[H_{\Lambda U^{1}}\right]^{-}} \leq\|\tilde{\beta}\|_{j^{\vee}}$ so $\mathfrak{j}^{\vee}$ is a subspace of $\mathfrak{j}^{\vee}\left[H_{\Lambda^{U^{1}}}\right]^{-}$. We shall denote the reproducing kernel of $j^{\vee}\left[H_{\Lambda^{U^{1}}}\right]^{-}$by $K_{\left[H_{\Lambda} u^{1}\right.}^{-}$. For this kernel, we obtain a similar result by exchanging + and - in (1).

\section{References}

[1] D. Alpay, Reproducing Kernel Spaces and Applications (2000), Springer Bassel AG.

[2] N. Aronszajn, Theory of Reproducing Kernels, (1950) Transactions of the American Mathematical Society Vol 68, No.3.

[3] H. Brezis, Functional Analysis, Sobolev spaces and partial differential equation (2011), Springer, pp.53-56, p.357.

[4] A. Cap, Lie Algebras and Representation Theory, Spring Term (2009), institut fur Mathematik, Universitat Wien, Nordbergstr. 15, 1090 Wien. pp. 13-72.

[5] P. Cyril, Aubin-Frankowski, Noyaux reproduisants d'Aronszajn et des mecaniques classique et quantique (2018). HAL, hal-01533963, pp. 4-10.

[6] J. Humphreys, Introduction to Lie Algebras and Representation Theory (1972), Third printing revised, Springer-Verlag, New York Heidelberg Berlin, pp. 1-24.

[7] P. Jorgenssen, F. Tian, Reproducing Kernels and choices of associated features spaces, in the form of $L^{2}$ - spaces (2017). Departement of Mathematics, The University of Iowa, Iowa City, Arxiv:2017.08492v1, pp. 2-4.

[8] J.H. Manton, P-O. Amblard, A Primer on Reproducing Kernel Hilbert Spaces. NOW the essence of knowledge (2015), pp. 9-19, pp. 34-44.

[9] A. Sagle, R. E. Walde, Introduction to Lie groups and Lie algebras. Academic Press (1973) New-York.

[10] S. Saitoh, Integral transforms, reproducing kernel and their applications. Pitman research notes in Mathematics (1997), pp. 20-27, pp. 37-39, pp. 47-53.

[11] S. Saitoh, Y.Sawano, Theory of reproducing kernels and Applications (2016), Developments in Mathematics Volume 44, Springer, pp. 1-10, pp. 37-39, pp. 47-53, pp. 86-88.

[12] P. Tauvel, R.P.W. Yu, Lie Algebras and Algebraic Groups (2005). Springer Monographs in Mathematics, p.299-318.

[13] S. Twareque Ali, J-P Antoine, J-P Gazeau, Coherent states, Wavelets and their Generalisations (2000). Graduate Texts in Contemporary Physics, Springer, pp. 89-106.

[14] I. Vern Paulsen and Mrinal Raghupathi, An introduction to the theory of reproducing kernel Hilbert spaces. (2016) volume 152 of Cambridge Studies in Advanced Mathematics. Cambridge University Press, Cambridge.

[15] G.Warner Harmonic Analysis on Semi-Simple Lie Groups I (1972). Die Grundlehren den mathematischen Wissenschatten in Einzeldarstellungen Band 188, pp. 3-10, p. 26. 